\title{
Hydrodynamic influence on the fractal morphology of the linoleic acid adsorbed layer at the mercury/electrolyte interface
}

\author{
D. Risović*, B. Gašparović ${ }^{+}$, B. Ćosović ${ }^{+}$ \\ *Molecular Physics Laboratory, ${ }^{+}$Center for Marine and Environmental Research, \\ Ruđer Bošković Institute, POB 180, HR-10002 Zagreb, Croatia
}

Fractal morphology of the adsorbed layer of linoleic acid has been studied for two adsorption regimes: diffusion controlled and mass-transfer controlled adsorption in a stirred solution.

The study has been conducted using ac voltammetry in combination with size scaling of the hanging mercury drop electrode for determination of the adsorbed layer fractal dimension.

It has been found that morphology of the linoleic acid adsorbed layer, as given by the fractal dimension, is a result of growth mechanism which is influenced by hydrodynamics (stirring or diffusion) and by the structure of the solution (monomers, dimers). At a lower fractional electrode coverage the adsorbed layer structure is primarily governed by the adsorption mechanism details. Fractal structure for high fractional electrode coverage is determined by geometrical constraints.

For the purely diffusion controlled adsorption process, the mechanism of the adsorbed structure growth is determined by the solution structure. This is manifested in fractal dimensions $D \approx 2.2$ and $\mathrm{D} \approx 2.5$, corresponding to the cluster-cluster and particle- 
cluster growth mechanisms, respectively. In a stirred solution, dominant influence on the growth mechanism of the LA adsorbed layer comes from shear, resulting in the same fractal dimension $(D=2.44)$ throughout the entire investigated concentration range (accumulation times).

\section{Introduction}

The extent of adsorption of an organic substance on electrode surface depends on its concentration, surface potential and molecular interaction. However, the adsorbed layer structure and its changes during the adsorption process may be also influenced by other parameters.

Adsorption of organic molecules has been studied by electrochemical methods for a long time. In regards to adsorption kinetics, several theoretical models have been developed [1-6]. They are divided in two classes; one assumes kinetic control of the process while the other assumes fast adsorption with diffusion controlled mass transport. In the case of diffusion controlled adsorption, it is generally assumed that the adsorbate surface concentration has the equilibrium value related to the bulk solution concentration far from the electrode. However, this is not always the case and the apparent isotherms are used to describe adsorption in such conditions. Adsorption processes can be studied in purely diffusion-controlled conditions or under stirring. In the case of mass transfer controlled adsorption in a stirred solution, the rate of adsorption is increased. The time-dependence of adsorption on a smooth mercury drop surface in a stirred solution was found [1]. The study of adsorption kinetics was 
also extended to kinetics of diffusion limited adsorption on not smooth but fractal electrode surfaces [7]. In general, hitherto more work has been directed towards studying adsorption on fractal surfaces [8-11] than fractality of the adsorbed layer itself or its changes during the adsorption process [12-14]. On the other hand, considerable theoretical [15-17] and experimental [18-20] efforts have been made to understand and to explain the fractal structures that result from processes of aggregation in two and three dimensions. As a consequence, it is a well-established fact that aggregates are fractal structures and that the magnitude of a fractal dimension is determined by the mechanism of aggregate growth [21-24]. A similar situation can be expected in the adsorbed layer formation.

If the rate of the adsorbed layer buildup is governed by the rate of mass transfer to the electrode, one may expect that the structure of the adsorbed layer will, at least to some degree, depend on the rate of the buildup. Therefore, the structure of the adsorbed layer should reflect the changes in the adsorption process induced by hydrodynamic influences. In turn, the change of adsorbed layer structure is reflected in the fractal properties (dimension) of the layer. Consequently, the hydrodynamic influences on the adsorption process can be inferred from fractal analysis of the adsorbed layer.

In previous paper we have developed a new method for determination of the adsorbed layer fractal properties. It has been demonstrated that in the case of the linoleic acid (LA) adsorption at the mercury electrode, subtle changes in the adsorption process resulting in structural changes of the adsorbed layer are reflected in, and can be observed through, the change in the corresponding fractal dimension [14]. Moreover, recently it was shown that geometrical features, such as fractality also reflect itself in basic material properties such as permittivity, polarization and specific 
capacity [25]. This indicates that fractal analysis could provide more subtle information of considered system than usually recognized.

Here, we have extended our study to the hydrodynamic influence on the development and morphology of the adsorbed layer of linoleic acid on the mercury/electrolyte interface. LA has an amphypathic molecular structure with the hydrocarbon tail responsible for hydrophobic interactions and the acidic group which participates in ionic interactions. These features make it suitable for this study. Namely, the adsorption kinetics and the structure of the adsorbed layer depend on the interactions between molecules themselves and with the surface, and on external forces being applied on them during the adsorption. In principle, such adsorption systems are described by the Frumkin isotherm that includes molecular interactions.

\section{Theory}

Generally, the fractal analysis approach is based on the possibility to describe quantitatively complex objects which are statistically scale-invariant, physical realizations of mathematical fractals that appear the same on all length scales. This property is manifested in a power-law dependence of the density-density correlation function $g(r) \sim r^{D-d}$, where $\mathrm{d}$ and $D$ denote spatial and fractal dimensions, respectively.

More generally, a power-law-scaling ratio characterizes one or more of the features of an object or a process carried out near or at the object:

$$
\text { Feature } \sim \text { scale }^{\Delta}
$$


Here "feature" should be considered in the broadest sense: it can be the surface area, scattered light intensity, the rate of heterogeneous reaction or the shape of adsorption isotherm. The "scale" can be particle size, pore diameter, scattering vector, cross-sectional area of an adsorbate or layer thickness.

The non-integer exponent $\Delta$, which indicates how sensitive the considered feature is to changes in the applied scale, has the meaning of dimension, which Mandelbrot [26] termed "fractal". Extension of this concept, coming from the recognition that effective geometries of various structures and processes can be described in terms of fractal geometry, results in an effective fractal dimension $D$ feature or simply $D$.

However, although Eq. (1) is generally valid, and often used, one must be cautious in its use and interpretation of power-law dependence as sign of fractality $[27,28]$. Because, mathematically speaking, power-law scaling is necessary but not sufficient condition. Self-similar fractals look similar at different scales, the property that leads to power-law scaling of various properties. However, the opposite is not true: power-low dependence of some feature on scale does not necessarily mean that the underlying structure is self-similar and that D has geometrical meaning. It is therefore necessary to verify assumptions of geometric self-similar structure and evaluate the cutoffs of scaling regime [29]. In spatial fractals, the scaling range is limited from below by the size of the basic building blocks composing the system, and from above with the system size. However, in practice this scaling range is usually further reduced due to system or apparatus limitations or properties.

Recently, we have established that the adsorbed layer of LA has a fractal structure [14]. That makes it a suitable testbed for our study because any hydrodynamical 
influence on the adsorption process and the structure of the adsorbed layer should be reflected in a change of its fractal properties.

To determine the fractal dimension of the adsorbed layer, we rely on the recently introduced method of capacitive current measurement in a system with size-scaling of hanging mercury drop electrode [14]. This method relies on relation (1) where the "feature" used is the capacitive current measured at a selected constant parameters (potential, $A C$ amplitude and frequency) and the "scale" is the surface area of the hanging mercury drop electrode represented through corresponding drop radius, $r$.

This is facilitated by the fact that the extent of adsorption of organic substances on the electrode surface is reflected in a change of differential capacitance of the electrode/solution interface, consequently in a change of measured capacitive current:

$$
i_{c} \propto C
$$

The simplest model of capacitive behavior of the electrode/solution interface is that of serially coupled capacitors [30], $\left(C_{1}\right.$ and $\left.C_{2}\right)$, representing spaces bounded by inner and outer Helmholtz planes. In such system, the layer with the lowest capacitance practically governs the total capacitance.

If organic molecules become adsorbed, this analogue circuit model is modified in such a way that a third capacitor, $C_{3}$, is added in parallel to the interface capacitance. $C_{3}$ represents the specific capacitance of the surface covered by organic molecules. Consequently, taking into account that $C_{1} \ll C_{2}$, the total specific capacitance is given by

$$
C \cong(1-\theta) C_{1}+\theta C_{3}
$$

here $\theta$ represents the fractional electrode surface coverage with organic adsorbates. 
Consequently, the total differential capacitance of the interface is given by

$$
C_{t}=(1-\theta) A C_{1}+\theta A C_{3}
$$

where $A$ is the area of electrode surface.

The capacitance $C_{3}$ is actually the sum of capacitors (formed at each adsorption site) randomly distributed over the electrode surface, and connected in parallel:

$$
C_{3}=\sum_{i} N_{i} C_{0 i}
$$

Here $C_{0 i}$ is the capacitance of single "molecular capacitor" and $N$ is the number of capacitors (adsorbed molecules) per unit area (in a monolayer, $\mathrm{N}$ is equal to the number density of adsorption sites).

These "molecular" capacitors have lesser capacitance than the original (empty) adsorption site due to lesser dielectric constant of adsorbed material and the increased separation of plates in the corresponding parallel-plate condenser that is proportional to the dimension of adsorbed molecule. Hence, due to this replacement of "original" capacitors at each adsorption site with the "molecular" capacitor of lesser capacitance, the overall capacitance decreases in proportion to the adsorbed material.

If we assume that the adsorbed molecules form a fractal structure, then, the number of molecules (“capacitors"), $n$, within any space of size $R$ is $n(R) \sim R^{D}$, where $\mathrm{D}$ is the fractal dimension. The average density of molecules (number of molecules per unit surface or volume) is given by:

$$
N \propto n(R) / R^{d}
$$

where $\mathrm{d}$ is the dimension of a space containing considered fractal structure.

Hence, for a fractal structure of size, $R$, the (surface) density, $\mathrm{N}$, varies as:

$$
N \propto R^{D-2}
$$


As the size (extent) of the adsorbed structure is determined by size of the electrode surface area that is proportional to the radius $r$ of the spherical drop, it follows that:

$$
N \propto r^{D-2}
$$

Since $C_{t} \sim N A$ and $A \propto r^{2}$ it follows that $C_{t} \propto r^{D}$ and consequently (cf. Eq.2):

$$
i_{c} \propto r^{D}
$$

or expressed through spherical electrode surface area:

$$
i_{c} \propto A^{D / 2}
$$

The same relation can be derived using the recent finding [25] that the specific capacitance, $C_{s}$, of any fractal structure scales as $C_{s} \sim R^{D-d}$. Now, the total capacitance is given by $C_{t}=A C_{s}$, and as $A \sim R^{2}$, taking into account Eq.2, we again get Eq. 6 .

Hence, the fractal dimension $D$ can be obtained from the slope of the log-log plot of $i_{c}$ vs. $A$

Here, it is worthwhile to point out that in our case the considered (fractal) system is the interface comprising the electrode surface with (or without) the adsorbate. If the electrode is scaled (expanded) the system scales accordingly.

If the electrode surface is smooth ("clean"- with no adsorbate present), the current should scale linearly with the area, i.e. $i_{c} \sim r^{2}$. In this case, the fractal dimension equals the classical Euclidean dimension $(D=2)$. However, if the surface morphology is changed from "smooth and uniform" to "irregular", but self-similar, this will be reflected in a different fractal dimension. Hence, since small mercury drop represents an ideally smooth and spherical surface the presence of the molecular layer with the fractal structure adsorbed at the surface will be reflected in the change of the fractal dimension. As the structure of the mercury drop surface remains unchanged during the experiment (it remains smooth) it is the change of adsorbed layer structure that 
causes the changes in fractal dimensionality of the system. For given molecular species and adsorption conditions, the structure of a fractal adsorbed layer is not different for different drop sizes. The adsorbed layer is not thicker, but only of larger surface size. It is self-similar with the same fractal dimension regardless of the drop size. This self-similarity of adsorbed layer, independent of system scaling, as revealed through the corresponding fractal dimension is the key assumption of our analysis.

Moreover, the fractal dimension is not only a useful descriptor of geometrical features of a certain structure, but it also provides information about the mechanism of structure growth. Computer simulations of aggregate growth in two and three dimensions [15-17] and experimental studies using Small Angle Neutron Scattering, Quasi Elastic Light Scattering [18-20] indicate that the magnitude of a fractal dimension is determined by the mechanism of aggregate growth. Aggregates formed through particle-cluster aggregation have fractal dimensions in the range 2.5-3.0 [21] while cluster-cluster aggregation results in lower fractal dimensions, typically 1.6-2.2 [22]. In the latter case, depending on particle stickiness, two different regimes of aggregation, resulting in a different fractal dimensions, can be distinguished. These are the diffusion-limited aggregation (DLA) and reaction limited aggregation (RLA), resulting in typical fractal dimensions around 1.8 and 2.1, respectively [23]. This suggests that the fractal dimension can potentially identify the aggregate formation mechanism as well as the stickiness (collision efficiency) of aggregating particles. 


\section{Experimental Section}

To study the fractal morphology of the adsorbed linoleic acid layer, phase sensitive ac voltammetry $\left(90^{\circ}\right.$ out of phase $)$ was used. Ac voltammetric measurements were performed by AUTOLAB with PGSTAT 20 (Ecochemie, Netherlands). Frequency of the ac voltage was $170 \mathrm{~Hz}$, amplitude $0.010 \mathrm{VMS}$, step potential 0.0021 $\mathrm{V}$, interval time $0.29 \mathrm{~s}$ and modulation time $0.19 \mathrm{~s}$. All experiments were performed in a three-electrode system with a hanging mercury drop electrode produced by Metrohm (Switzerland). $\mathrm{Ag} / \mathrm{AgCl} / 3 \mathrm{M} \mathrm{KCl}$ electrode was used as the reference electrode and a platinum wire as the auxiliary electrode. Measurements were done in a potential range of $E=-0.35 \mathrm{~V}$ to $-0.90 \mathrm{~V}$, in which there is no desorption of LA. The results were elaborated for the electrode potential $E=-0.6 \mathrm{~V}$, approximately representing a non-polar electrode. At this potential there are no oxido-reduction processes of included molecular species present in the investigated system.

The study of the adsorbed LA layer fractal morphology was conducted for a solely diffusion controlled adsorption process and mass transfer controlled adsorption in a stirred solution (270 r.p.m). For the solely diffusion controlled process, accumulation times were in range from $30 \mathrm{~s}$ to $7 \mathrm{~min}$ in the corresponding LA concentration range of $0.04-30 \mathrm{mg} / \mathrm{dm}^{3}\left(1.410^{-7}-1.0710^{-4} \mathrm{M}\right)$. A secondary set of measurements were done in solutions under stirring and for accumulation times ranging from $20 \mathrm{~s}$ to $3 \mathrm{~min}$ in the concentration range of $0.006-70 \mathrm{mg} / \mathrm{dm}^{3}\left(1.410^{-7}\right.$ $\left.2.510^{-4} \mathrm{M}\right)$

For the purpose of fractal analyses, in voltammetric measurements the volume of the mercury drop electrode was varied stepwise over nine sizes in the range of 0.08 to 
$0.7 \mathrm{~mm}^{3}$, which corresponds to the electrode surface area in the range of 0.9 to 3.8 $\mathrm{mm}^{2}$.

For every investigated concentration of linoleic acid, voltammetric measurements were performed with nine different drop sizes, each time with a freshly prepared new drop of a selected size. The drop size was not changed during one voltammetric measurement.

Linoleic acid (Fluka, Switzerland) was used without further purification. Mercury was purified by double distillation under reduced pressure. $\mathrm{NaHCO}_{3}$ (Merck) was used without prior purification. $\mathrm{NaCl}$ (Kemika, Croatia) was purified by prolonged heating at $450^{\circ} \mathrm{C}$. All solutions were prepared with deionised water obtained with the Milly-Q Water System (Millipore, Switzerland). Carbonate buffer was used to maintain $\mathrm{pH}$ 8.3. All solutions simulated seawater composition $\left(0.5 \mathrm{M} \mathrm{NaCl}, 2 \times 10^{-3}\right.$ $\mathrm{M} \mathrm{NaHCO}_{3}$, and $\mathrm{pH} 8.3$ ).

\section{Results and Discussion}

The fractal dimension of the adsorbed layer was estimated from voltammetric measurements conducted under the conditions of strictly diffusion controlled adsorption and mass transfer controlled adsorption in a stirred solution. Differential capacity curves vs. applied potential and vs. LA concentration are presented in our previous paper [14]. 
Diffusion controlled adsorption. Changes of the fractal dimension of the adsorbed LA layer were measured for seven accumulation times in a broad concentration range of LA. Typical changes of $D$ vs. LA bulk concentration obtained for 1 and $5 \mathrm{~min}$ accumulation times are presented in Figure 1a, while the corresponding apparent adsorption isotherms are given in Figure 1b. Other curves for different accumulation times are not included for the sake of clarity, but maximal Dvalues are depicted in Figure 1a.

It can be seen from Figure 1a that the fractal dimension changes with an increase in the LA bulk concentration in a similar manner regardless of accumulation time. The same was obtained for the other accumulation times used. A distinct main (highest) peak along with few less pronounced pre- and post- peaks characterizes the changes of $D$ in the investigated LA concentration ranges.

The main concern of this study is the main fractal peak. The value of this peak provides information on the mechanism of the adsorbed layer formation. Although the main peak for different accumulation times appears at different bulk concentrations, these always correspond to the fractional electrode coverage of about $70 \%$, as inferred from the apparent adsorption isotherms (Figure 1b). To attain certain fractional electrode coverage in shorter accumulation times, higher LA bulk concentrations are needed than for longer accumulation times. Hence the observed shifts of peak position toward the lower LA concentration with increased accumulation time in the $D$ vs. concentration graph.

Dependence of the maximal D-value of the LA adsorbed layer on accumulation time is shown in Figure 2 (curve 1), along with the corresponding fractal dimension values for pure electrolyte, obtained under appropriate experimental conditions (curve 2). The overall average fractal dimension for pure electrolyte 
$D=1.99 \pm 0.02$ corresponds to the spatial dimension, reflecting a smooth electrode surface without any adsorbed substance.

Curve 1 in Figure 2 representing the change of maximal- $D$ has a sigmoidal form: two nearly constant value ranges connected with a transition region. $D$ changes from $D=2.20$, obtained for shorter adsorption times (higher bulk concentration), and increases toward $D=2.52$ at longer adsorption times, indicating that the corresponding bulk concentration, related to the selected accumulation time, plays an important role in the formation and growth of the adsorbed layer and its structure.

We suppose that different mechanisms of layer growth are responsible for the layer formation in the case of low bulk concentrations (longer adsorption times) and in the case of higher bulk concentrations (short adsorption time).

It is well known that fractal properties are directly related to the growth mechanism of a given fractal structure [21-23]. Hence, the observed behavior can be explained within the theoretical framework of the fractal structure growth mechanisms.

In our case, molecules contributing to the growth of the adsorbed layer are transported by means of random diffusional walks (Brownian movement). For a given mechanism of structure formation, theoretical modeling predicts a single class of fractal objects with well-defined exponents. To identify the growth process responsible for the observed fractal behavior, mathematically strict identification requires a large range of scale invariance (well over one order of magnitude), [31,32] which is seldom or never found in adsorption measurements. Hence, the limitation of the fractal invariance scale to only one order of magnitude results in uncertainty of the inferred fractal dimension [24]. This uncertainty results in a "smeared" fractal dimension instead of the single precise value predicted by theory. 
For shorter accumulation times (30 sec and $1 \mathrm{~min})$, the observed maximal- $D$ is $\approx 2.2$. Theoretically, such a fractal dimension is obtained from the so-called ghost models for the case of cluster-cluster three-dimensional aggregation [33]. For such a fractal structure growth mechanism, the hierarchical ghost models (with volume correction excluded) predict $D=1.6-2.2$ [34] and the non-hierarchical reactionlimited aggregation in three dimensions gives $D=2.2$ [35]. This result was obtained for the cluster- cluster aggregation of clusters of equal mass (size). In reality there are size (mass) distributions which, when taken into account, result in a slight rise of $D$ [36].

In our case, higher LA bulk concentrations $\left(\geq 3.210^{-6} \mathrm{M}\right.$ or $\left.0.9 \mathrm{mg} / \mathrm{dm}^{3}\right)$ are required to reach the maximal peak value of $D$ in a short adsorption time. Hence, on the basis of the observed maximal $D$ and considering the above-discussed theoretical models, we concluded that the aggregation of LA molecules had already occurred in the bulk solution. This is in agreement with the observation that even below CMC (critical micellar concentration) fatty acids exhibit a strong tendency to form associates, mainly dimers [37,38]. For linoleic acid, the dimerization constant, $\mathrm{K}_{\mathrm{D}}$, is $4.0 \times 10^{6}\left(\mathrm{dm}^{3} / \mathrm{mol}\right)$, indicating that linoleate ions are present in the dimeric form even at a concentration of $10^{-6} \mathrm{M}\left(0.28 \mathrm{mg} / \mathrm{dm}^{3}\right)$. Such "clusters" (dimers), formed in the bulk solution, adsorb on the electrode surface, interacting with the already adsorbed clusters and forming a fractal structure with the maximum value of $D=2.2$. This structure occurs at the fractional electrode coverage of $\approx 70 \%$.

For longer accumulation times ( $\geq 5 \mathrm{~min}$ ), the $70 \%$ fractional electrode coverage is attained at lower bulk concentrations $\left(<0.35 \mathrm{mg} / \mathrm{dm}^{3}\right)$ than for short accumulation times. At a low bulk concentration, the majority of the molecules are monomers so that molecules arrive at the electrode surface one at a time. Here they 
join the already adsorbed molecules or clusters. In theory such process is known as particle-particle or particle-cluster fractal structure growth (aggregation).

The above mentioned processes can be classified according to how an incoming particle moves to join the cluster [34.39]. If a particle joins the structure via random walk, tenuous fractal structure results with the fractal dimension lower than the corresponding Euclidean space dimension $(D<d)$. Diffusion-limited aggregation (DLA) models give $D=2.5$ for $d=3$.

There is a transition region between the low and high LA concentration ranges characterized by predominantly monomer or dimer forms in solution. In the transition region, two concurrent mechanisms of layer formation are present due to the binary character of the bulk solution, containing monomers and dimers in various ratios. Hence, in this region the fractal dimension reflects this composition and is $2.2<D<2.5$.

Regardless of the mechanism involved, the overall process of the layer growth and transformation is similar for different accumulation times. At a low fractional electrode coverage the process is more susceptible to the adsorption process details, determined by the conditions in the bulk, while at a high fractional coverage the geometrical constrains become significant. These constraints lead to a decrease in the fractal dimension, approaching values of $D=1.95-1.98$. Hence, the maximal fractal dimension of the developed layer structure depends on the conditions in the bulk, which determine the growth mechanism.

These results are in agreement with the earlier experimentally observed differences in studies of inhibition of the Cd(II) electrode reaction in the presence of the adsorbed layer of nonionic surface active substance Triton-X-100 from solutions of different bulk concentrations [40]. This difference was attributed to the adsorption 
of dimers and/or association processes that occurred at the electrode surface for solutions of higher bulk concentrations.

Mass-transfer controlled adsorption in a stirred solution. Changes of the adsorbed layer fractal dimension in dependence on the LA bulk concentration in a stirred solution were measured for four accumulation times. Figure 3 presents typical changes of $D$ vs. LA bulk concentration obtained for 1 and 3 min accumulation times. Maximal $D$-values for other investigated accumulation times are also depicted. The change of $D$ in the LA concentration range investigated is characterized by distinct main (highest) peak and a few less pronounced peaks similarly to those previously observed for diffusion controlled adsorption.

Figure 4 shows that the maximal fractal dimension is almost independent of the investigated adsorption time. The average fractal dimension is $\bar{D}=2.44 \pm 0.02$.

The obtained result can be explained by considering the influence of fluid motion, in addition to the Brownian movement, on the mechanism of the adsorption process. This situation is rather complex because there are several relevant parameters that are modified during stirring. Consequently, interaction processes between molecules (particles) are modified both in the bulk and near the adsorption interface, leading to different fractal behavior with regard to diffusion controlled adsorption. These modifications include the preferred aggregation mechanism in the bulk and also its changes induced by changes in the shear rate, modification of the Brownian trajectory and changes in the diffusion tensor components of the approaching molecules (particles) near the interface.

As regards the situation in the bulk, it was shown that cluster-cluster $(\mathrm{Cl}-\mathrm{Cl})$ aggregation is the dominating mechanism of coagulation in a shear flow [41]. This is 
inferred from purely hydrodynamic considerations of capture (sticking) efficiencies at collision, which are much higher for particles of similar sizes (cluster-cluster or particle) than between particles of different sizes (particle-cluster). Namely, for a particle of size $\boldsymbol{a}$ (moving along an open streamline) to be captured, it must come to a distance $\leq \boldsymbol{a}$ of the aggregate. However, the distance to the nearest open streamline is $0.16 R$ (for a compact aggregate of radius $R$ ); hence, since $R>>\boldsymbol{a}$, a small particle cannot be captured by a bigger aggregate. Hence, once the clusters are formed in the bulk, it is more probable that the aggregate growth in the stirred solution will continue by cluster-cluster than by molecule-cluster association. Cluster-cluster aggregation mechanism is associated with $D \approx 2.2$; hence, that is a fractal dimension which may be expected in the considered situation. However, there are some additional shear effects that should be included that have a significant impact on cluster formation. Namely, the viscous shear stress generated during the stirring influences the fractal structures in the bulk and can even cause a structure break-up [41,42]. It was shown that, with an increase of the dimensionless shear rate $G$, a contraction of fractal structure occurs, and that a certain maximum structure density and size exist. As a consequence, a critical fractal dimension is attained at some $G=G_{\text {critical }}$. At $G>G_{c r i t i c a l}$, further contraction of the structure is impossible (it remains rigid) and its eventual break-up can be attributed to mechanical stresses. Sonntag and Russell ${ }^{42}$ have shown that the fractal dimension of aggregates for Brownian coagulation without shear deformation in accordance with the cluster-cluster aggregation mechanism is indeed 2.2. But, when shear was applied fractal dimension increased up to $D_{\text {critica }}=2.5$ and then remained constant; i.e. the aggregates become rigid and no further compaction was observed. In a more detailed study of this problem Potanin [41] estimated $D_{\text {critica }}=2.42$. 
On the basis of this consideration and due to increased collision frequency, we may expect that in our experimental conditions more dimers will be present in the bulk for a given concentration range than under equivalent conditions of an unstirred solution. This situation would be also reflected in the structure of the adsorbed layer.

However, although the average fractal dimension $\bar{D}=2.44 \pm 0.02$ obtained in our experiment for all investigated accumulation times is in perfect agreement with $D_{\text {critical }}$ obtained by Potanin [41], we do not believe that the described compaction mechanism is fully responsible for it. Firstly, most of our investigations with stirring are done in solutions of relatively low concentrations. Secondly, LA is not a big molecule and is not likely to produce large aggregates, at least in the investigated concentration range. As we have stated earlier, to investigate much higher concentrations (at $\theta \approx 0.7$ ) would require extremely short accumulation times, which are connected with experimental difficulties and substantially higher measurement uncertainties. Thus, we do not expect the described processes $(\mathrm{Cl}-\mathrm{Cl}$ aggregation and hydrodynamical compaction) to be dominant in the bulk. However, they could, to some extent and for short accumulation times, play a certain role in the diffusive layer near interface.

To explain the obtained fractal dimension $D \approx 2.44$ and its observed invariability with accumulation time, some additional effects should be taken into consideration. Particularly those which influence adsorption at the interface.

Brownian motion and shear flow have a coupling effect on collision processes in stirred solutions. A small amount of shear distorts the isotropic situation of diffusion, and a small amount of Brownian motion transfers the molecule (particle) to a different trajectory, bringing about different collision efficiency. Brownian motion is dominant for $\mathrm{Pe}<1$ and shear is large if $\mathrm{Pe}>100$. Pe is the translational Peclet 
number, denoting strength ratio between fluid flow and diffusivity of particles. In particular, hydrodynamics influences the diffusion tensor of the particle approaching the adsorption plane. Whereas in the bulk the diffusion tensor is isotropic and reduces to scalar (diffusion coefficient), near the adsorption plane the parallel components of diffusion tensor decrease more slowly than in the perpendicular component. As a consequence, the molecule (or particle) can diffuse extensively parallel to the plane during the time needed to reach the adsorption plane. Thus, hydrodynamic interactions tend to allow the adsorbing particles (molecules) to randomize their position over the available space before they adsorb. Consequently, this would lead to the densely packed structure having a higher $D$. This situation is similar to the one encountered in the random sequential adsorption model $[43,44]$.

On the other hand, it was observed in DLA that the fractal dimension of cluster (aggregate) depends on the fractal dimension of the diffusive trajectory $\left(d_{w}\right)$ of the approaching particle. As observed in the particle-cluster and cluster-cluster aggregation fractal dimension $(D)$ increases when $d_{w}$ decreases [34,39]. Thus, for the purely Brownian trajectory $d_{w}=2$ and for ballistic $d_{w}=1$, hence ballistic-growth models predict densely packed structures with $D \approx \mathrm{d}$. If the real viscosity of the fluid is included a modified ballistic aggregation model results, which permits less dense structures $(\mathrm{D}<\mathrm{d})$ to be formed [17]. Also, the presence of electrolyte in combination with the stirring induced hydrodynamical effects influences the aggregation process and gives rise to a fractal dimension of $\mathrm{D}=2.4[45]$. The same fractal dimension was found for activated sludge flocks [46].

Thus, the foregoing points to the conclusion that in the case of a stirred solution the change of the approaching particle's diffusion tensor components near the electrode surface leads to a lower fractal dimension of its trajectory and consequently 
to a higher fractal dimension of the adsorbed structure than expected at higher bulk concentrations.

Accordingly, we may conclude that the growth mechanism of the LA adsorbed layer from stirred solution is principally determined by shear and not by bulk concentration, resulting in the same maximal fractal dimension $(D \approx 2.44)$ throughout the entire investigated concentration range (accumulation times).

\section{Summary and Conclusions}

Hydrodynamic influence on fractal properties of the adsorbed layer was studied using the recently introduced method of mercury drop electrode scaling for determination of the fractal dimension of the adsorbed layer [14]. The underlying assumption is that although the structure of the adsorbed layer may change, as long as it remains self-similar, hence scale independent (or fractal) it may be characterized with corresponding fractal dimension directly correlated with its structure.

The fractal dimension of adsorbed layer changes during the adsorption process, reflecting the different stages of adsorption. The maximal-fractal dimension is related to the dominant mechanism responsible for the adsorbed layer growth. In regard to accumulation time, for longer accumulation times, the maximal- $D$ value appears at a lower LA bulk concentration. Conversely, for shorter accumulation times this value appears at higher LA bulk concentration. However, this peak value is always observed at the fractional electrode coverage of about $70 \%$. 
The morphology of the adsorbed layer, characterized by the fractal dimension value, is the result of the growth mechanism which, according to our observations, is influenced by hydrodynamics and by the structure of the solution.

For the purely diffusion controlled adsorption process, the mechanism of the adsorbed structure growth is determined by the structure of the solution (monomers, dimers). Regarding the solution structure, there are two border cases characterized by two distinct mechanisms corresponding to the solutions in which the molecules are predominantly in the monomeric (lower bulk concentrations) or dimeric (higher bulk concentration) form. Between these two states (concentration regions) there is an intermediate state of solution where no form dominates and where two concurrent growth mechanisms are present. Theoretically, these mechanisms are described within the framework of the diffusion limited aggregation (DLA) model.

At lower LA bulk concentrations where LA molecules are mostly in the form of monomers, experimentally corresponding to a long accumulation time, the maximal-fractal dimension of the adsorbed layer is 2.5 . This indicates the particlecluster DLA.

Fractal dimension $D=2.2$, obtained for the higher LA bulk concentration (short accumulation times), corresponds to the cluster-cluster DLA. It indicates that dimers are formed in the solution and as such approach and attach to the electrode surface and adsorb.

For intermediate concentrations, concurrent growth mechanisms result in the $D$ reflecting monomer-dimer ratio in the bulk.

For a stirred solution, due to a hydrodynamic modification of random (Brownian) walk and competitive shear induced structure compaction, in the investigated concentration range the maximal-fractal dimension of the adsorbed layer 
is practically independent of the bulk concentration, and equal to $D \approx 2.44$. We identify this fractal dimension as corresponding to the shear controlled growth mechanism. This is justified by the fact that many other investigations conducted in considerably different experimental conditions and systems in the presence of shear flow resulted in the same fractal dimension.

In conclusion, these results point to the fact that different fractal structures of the adsorbed layer are obtained for different experimental conditions (stirring, accumulation time). At a high fractional electrode coverage, these effects are less pronounced due to the geometric constraints, which become the dominant growth factor. However, at a lower fractional electrode coverage these effects should be considered because they may lead to the formation of adsorbed layers of different densities and structures. Hence, such considerations might be important for the studies in which the layer structure morphology plays a significant role, e.g. incorporation of relevant molecules in monolayers, studies of biological membranes or capacitance of "small systems" (micellar, biological, polyelectrolite, etc.).

Acknowledgement: The authors acknowledge support from the Croatian Ministry of Science and Technology grants No. 0098029 and No. 0098122. 


\section{References}

[1] P. Delahay, I. Trachtenberg, J. Am. Chem. Soc. 79 (1957) 2355.

[2] P. Delahay, D.M. Mohilner, J. Am. Chem. Soc. 84 (1962) 4247.

[3] R. Hansen, J. Am. Chem. Soc. 82 (1960) 637.

[4] W.H. Reinmuth, J. Phys.Chem. 65 (1961) 473.

[5] J.D. Tirado, D. Acevedo, R. L. Bretz, H.D. Abruna, Langmuir 10 (1994) 1971.

[6] K.L. Filippov, J. Colloid Interface Sci. 182 (1996) 3330.

[7] A. Sery-Levi, D. Avnir, J. Phys. Chem. 97 (1993) 10380.

[8] T. Pajkossy, J. Electroanal. Chem. 300 (1991) 1.

[9] P. Pfeifer, New. J. Chem. 14 (1990) 221.

[10] D. Avnir, D. Farin, P. Pfeifer, New. J. Chem. 16 (1992) 439.

[11] M.W. Cole, N.S. Holter, Phys. Rev. B 33 (1986) 8806.

[12] T. Wandlowski, L. Pospíšil, J. Electroanal. Chem. 270 (1989) 319.

[13] L. Pospišil, J. Phys. Chem. 92 (1988) 2501.

[14] D. Risović, B. Gašparović B. Ćosović, Langmuir 17 (2001) 1088.

[15] P. Meakin, F. Family, Phys.Rev. A 36 (1987) 5498.

[16] R.D. Mountain, G.W. Mulholand, Langmuir 4 (1988) 1321.

[17] J.F. Dirkse, J.D. Cawley, J.Colloid Interface Sci. 170 (1995) 466.

[18] D.W. Schaefer, J.E. Martin, P. Wiltzius, D.S. Cannell, Phys. Rev. Lett. 52 (1984) 2371.

[19] D.A. Weitz, M.Y. Lin, Phys. Rev.Lett. 57 (1986) 2037.

[20] J.E. Martin, Phys. Rev. A 36 (1987) 3415.

[21] D.W. Schaefer, Science 243 (1989) 1023.

[22] T.A. Witten, M.E. Cates, Science 232 (1986) 1607. 
[23] P. Meakin, Adv. Colloid Interface Sci. 28 (1988) 249.

[24] M. Kolb, J. Non-cryst. Solids 121 (1990) 227.

[25] D. Risović, B. Gašparović B. Ćosović J. Chem. Phys. 106 (2002) 9810.

[26] B.B. Mandelbrot, The Fractal Geometry of Nature; Freeman: San Francisco, 1982.

[27] D. Hamburger, O.Biham and D. Avnir, Phys. Rev. E, 53 (1996) 3342.

[28] O. Malcai, D.A. Lidar, O. Biham, Phys. Rev. E 56 (1997) 2817.

[29] D. Avnir, O. Biham, D. Lidar, O. Malcai, Science 279 (1998) 39.; B. Mandelbrot, ibid. p .783. P. Pfeifer, ibid. p.784, A.A. Tsonis, ibid. p .787

[30] J.O’M. Bockris, M.A.V. Devanathan, K. Müller, Proc. R. Soc. London A, 274 (1963) 55.

[31] D. Avnir, D. Farin, P. Pfeifer, Nature 308 (1984) 261.

[32] D. Avnir (Ed.), The fractal Approach to Heterogenous Chemistry: Surfaces, Colloids, Polymers, Wiley, Chichester,1989; reprinted 1990.

[33] R.C. Ball, T.A. Witten, J. Stat.Phys. 36 (1984) 863.

[34] R. Julien, M. Kolb, J.Phys. A: Math. Gen. 17 (1984) L639.

[35] W.D. Brown, R.C. Ball, J. Phys. A 18 (1985) L517.

[36] R.C. Ball, Physica B 127 (1984) 87.

[37] P. Mukerjee, J. Phys. Chem. 69 (1965) 2821.

[38] P. Mukerjee, Advan. Colloid Interface Sci. 1 (1967) 241.

[39] P. Meakin, Phys. Rev.A, 29 (1984) 997.

[40] Z. Kozarac, S. Nikolić, I. Ružić, B. Ćosović, J Electroanal. Chem. 137 (1982) 279.

[41] A.A. Potanin, J. Colloid Interface Sci. 145 (1991) 140.

[42] R.C. Sonntag, W.B. Russel, J. Colloid Interface Sci. 113 (1986) 399. 
[43] P. Schaaf, J-C. Voegel, B. Senger, J. Phys. Chem. B 104 (2000) 2204.

[44] J. Feder, J. Giaever, J. Colloid Interface Sci. 78 (1980) 144.

[45] S. Stoll, A. Elaissari, E. Pfefferkorn, J. Colloid Interface Sci. 40 (1990) 98.

[46] A. Thill, S.Veerapaneni, B. Simon, M. Wiesner, J.Y.Bottero, D. Snidaro, J. Colloid Interface Sci. 204 (1998) 357. 


\section{Figure captions}

Fig. 1. Diffusion controlled adsorption: (a) dependence of the adsorbed layer fractal dimension on the bulk concentration of linoleic acid solution for accumulation times: 1 min (curve 1) and 5 min (curve 2). Symbols denote measurement points, while lines represent the fit with B-spline For the clarity of presentation only several error bars corresponding to the standard deviation are added. Also are shown the maximal- $D$ values obtained for investigated accumulation times, $30 \mathrm{~s}, 90 \mathrm{~s}, 2 \mathrm{~min}, 3$ min and 7 min. (b) corresponding apparent adsorption isotherm for 1 (1') and 5 min (2') accumulation times. Symbols denote measurement points, while lines represent the fit with B-spline. Size of the symbols corresponds to experimental error

Fig. 2. Diffusion controlled adsorption: maximal value of the adsorbed layer fractal dimension vs. accumulation time (curve 1). The corresponding values of fractal dimension obtained for pure electrolyte under the same experimental conditions (curve 2). Symbols denote measurement points, while lines represent the fit with Bspline. Error bars correspond to the standard deviation.

Fig. 3. Mass transfer controlled adsorption in a stirred solution: (a) dependence of the adsorbed layer fractal dimension on bulk concentration of linoleic acid solution for the accumulation times: of $1 \mathrm{~min}$ (curve 1) and $3 \mathrm{~min}$ (curve 2). Also the maximal$D$ values obtained for investigated accumulation times of $20 \mathrm{~s}$ and $4 \mathrm{~min}$ are shown. Symbols denote measurement points, while lines represent the fit with B-spline. For the clarity of presentation only several error bars corresponding to the standard deviation are added. 
Fig. 4. Mass transfer-controlled adsorption in a stirred solution: maximal value of the adsorbed layer fractal dimension vs. accumulation time (curve 1), and the corresponding values of fractal dimension obtained for pure electrolyte under the same experimental conditions (curve 2). Symbols denote measurement points, while lines represent the fit with B-spline. Error bars correspond to the standard deviation. 
Fig. 1.

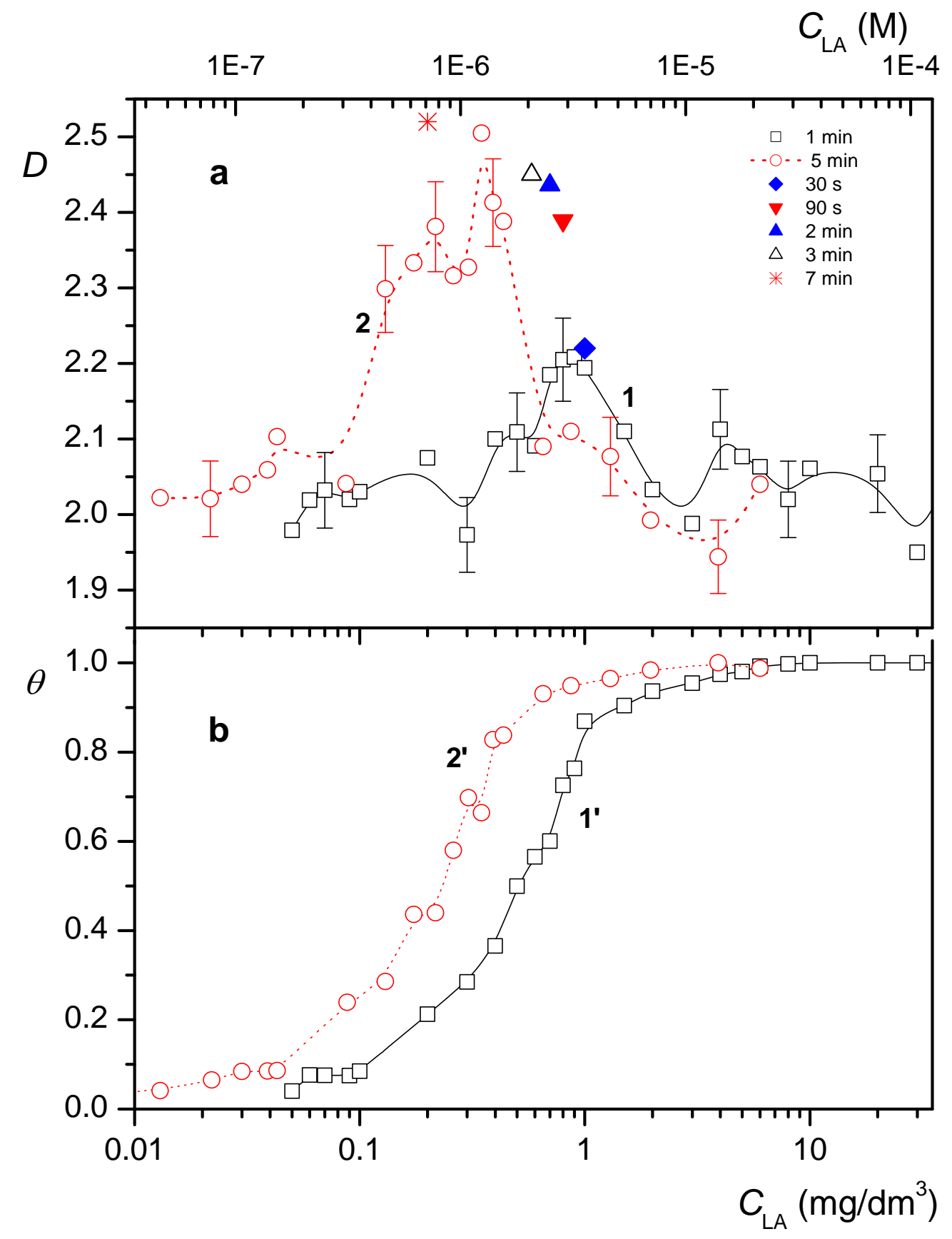


Fig. 2

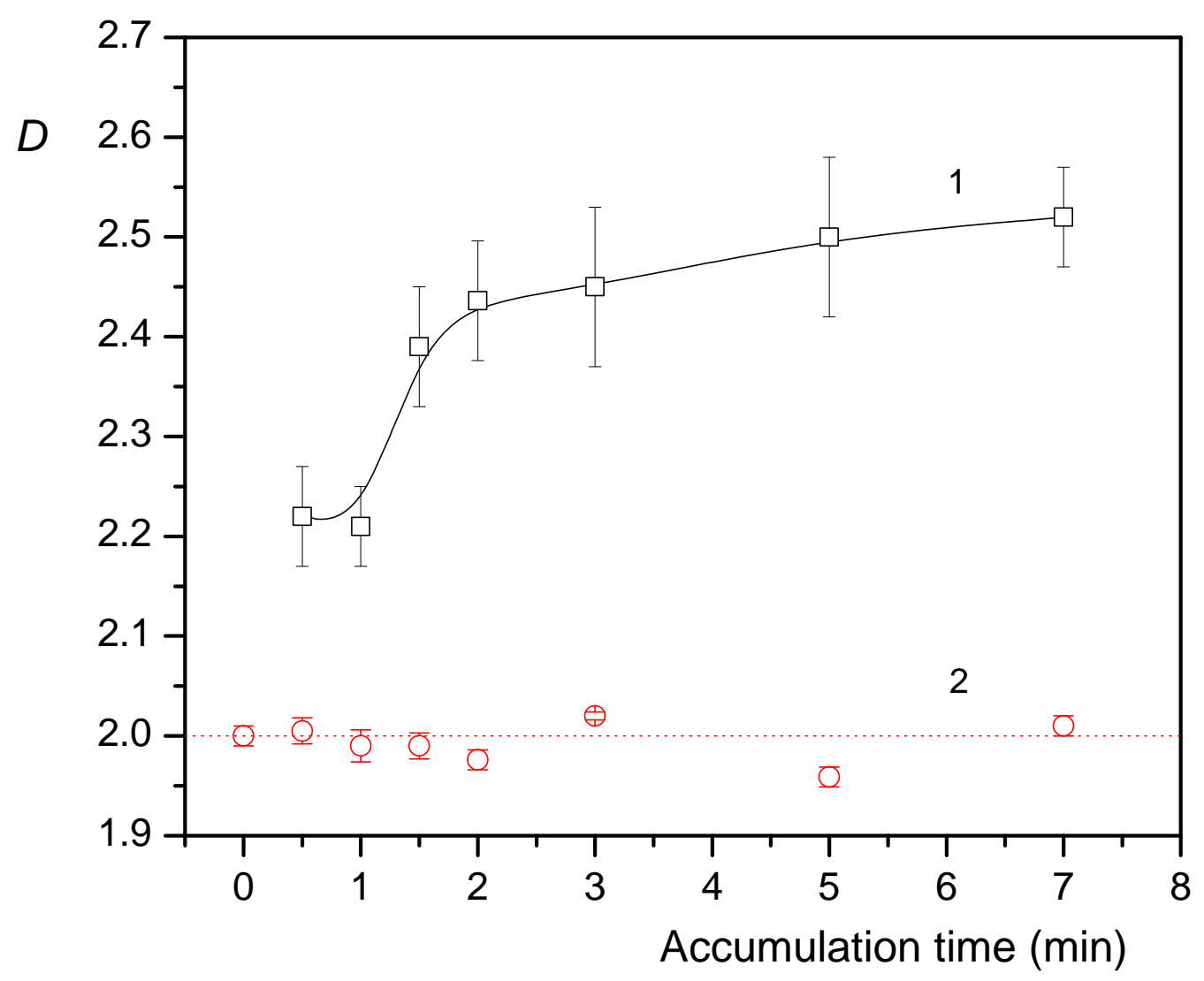


Fig. 3

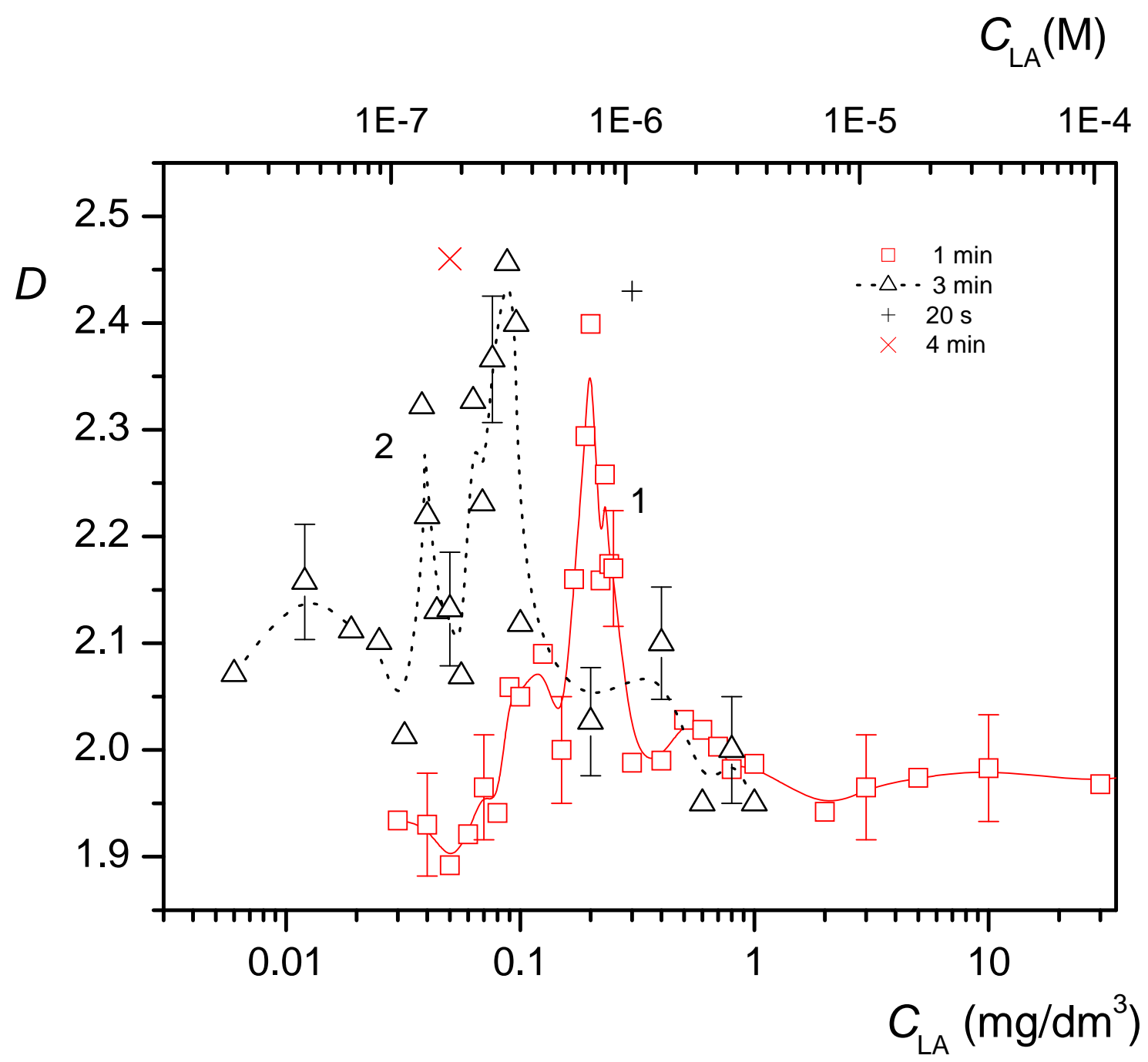


Fig. 4

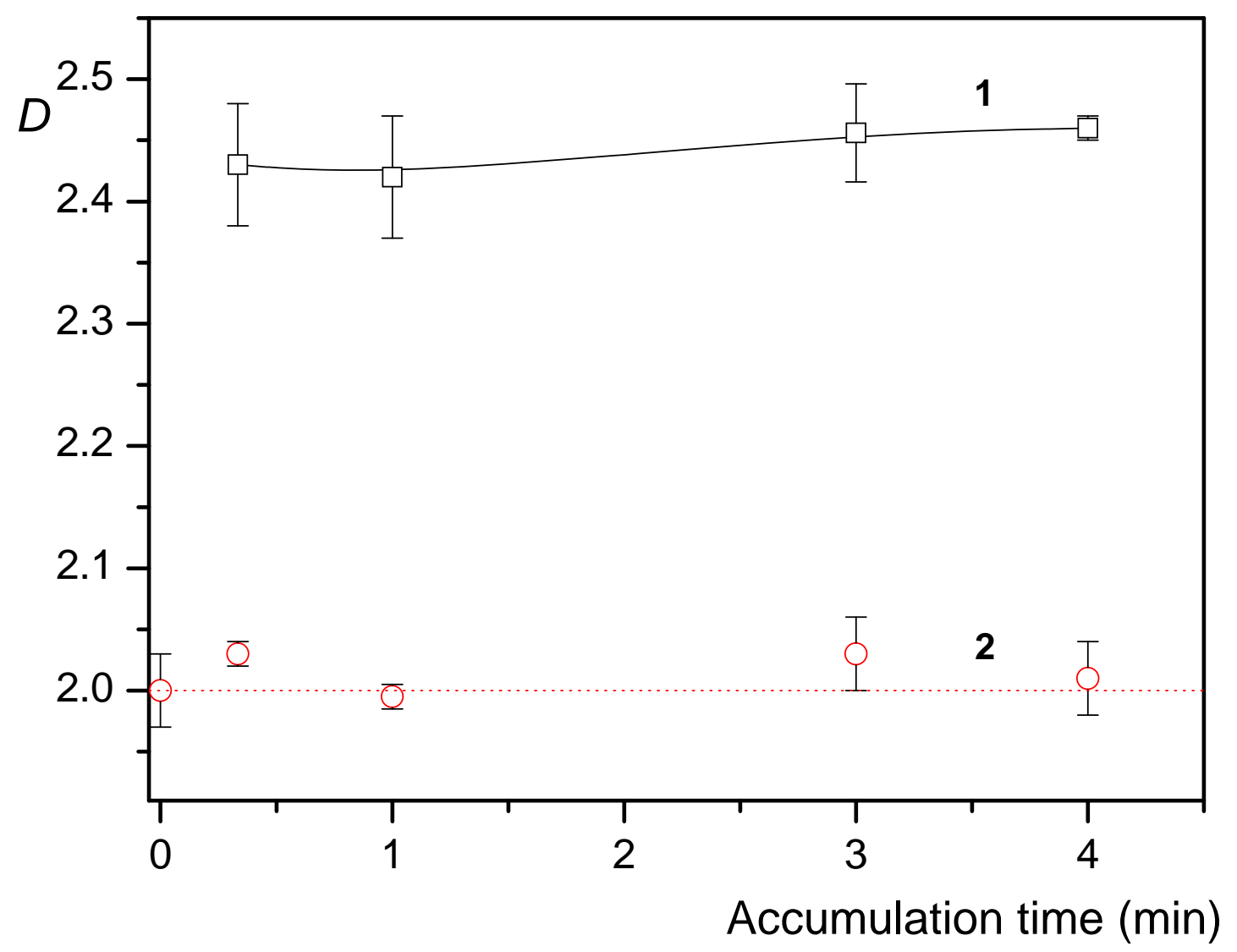

